\title{
A Low Score in Patients with a Positive PCA3 Urine Test Predicts the Lowest Prognostic Category in the New Grading System for Prostate Carcinoma
}

\author{
Rodon $\mathbf{N}^{1 *}$, Trias $\mathbf{I}^{1,2,3}$, Verdu $\mathbf{M}^{1,2}$, Calvo $\mathbf{M}^{3}$, Banus JM11,4 and Puig X $\mathbf{X}^{1,2,5}$ \\ 1BIOPAT, Biopatologia Molecular SL, Grup Assistència, Spain \\ ${ }^{2}$ HISTOPAT Laboratoris, Spain \\ ${ }^{3}$ Department of Statistics, Faculty of Biology, Universitat de Barcelona, Spain \\ ${ }^{4}$ ICUN, Institut Catalàd' Urologiai Nefrologia, Spain \\ ${ }^{5}$ Hospital de Barcelona, SCIAS, Grup Assistència, Spain
}

\section{Research Article \\ Volume 2 Issue 1}

Received Date: December 21, 2017

Published Date: January 08, 2018

*Corresponding author: Natalia Rodon, BIOPAT, Biopatologia Molecular SL, Avinguda Diagonal, 660. Planta -1.08034 Barcelona, Spain, Tel: +34 932030 837; Email: nrodon@biopat.es

\section{Abstract}

Purpose: In this study we evaluate the PCA3 as a tool to improve prostate cancer (PCa) screening and it's capability to predict PCa aggressiveness.

Materials and Methods: This retrospective study included data from consecutive patients with suspected PCa who presented to the urology office between November 2009 and April 2016 and were candidates for prostate biopsy. A total of 1038 urine samples were tested in our laboratory with a kit that generated a PCA3 score (s-PCA3). A prostate biopsy was recommended only in those patients with s-PCA3 $\geq 35$. Associations between variables were analyzed using the $\mathrm{R}$ software.

Results: In patients with a positive s-PCA3 (44.5\%), a subsequent biopsy was recommended. A total of 151 biopsies were studied, 56.3\% yielded a diagnosis of PCa. The probability of a positive biopsy increased as the s-PCA3 increased $(p=0.041)$. The percentage of affected cylinders increased as the $s$-PCA3 increased $(p=0.015)$. A statistically significant relationship was observed between s-PCA3 and both the Gleason score and the Grade Group ( $\mathrm{p}=0.001$ and 0.008 , respectively). The best log linear models and a logistic model confirmed the relationships shown previously with Fisher's exact tests.

Conclusions: s-PCA3 may serve as an additional marker to reduce the indication for biopsies and avoid overdiagnosis and overtreatment of patients with suspected PCa. The prognostic significance of s-PCA3 was confirmed, as it was associated with tumor volume and Gleason score. Importantly, to our knowledge this is the first time that an association has been demonstrated between s-PCA3 and the new Grade Group.

Keywords: Gleason Grade; Prostate Grade Group; PCA3; PSA; Screening 


\section{Open Access Journal of Cancer \& Oncology}

Abbrevations: PCa: Prostate Cancer; PSA: ProstateSpecific Antigen; BPH: Benign Prostatic Hyperplasia; ISUP: International Society of Urological Pathology; ASAP: Atypical Small Acinar Proliferation.

\section{Introduction}

Prostate cancer (PCa) is the second most common cancer and the fifth leading cause of cancer death in men worldwide [1]. PCa has been described as a heterogeneous disease with varying clinical and morphological characteristics [2]. Traditionally, screening has been based on digital rectal examination (DRE) and measurement of serum prostate-specific antigen (PSA), which has a low specificity for PCa [3-6]. The management of patients with altered PSA values not exceeding $10 \mathrm{ng} / \mathrm{ml}$, known as the PSA gray zone, is especially challenging because $75 \%$ of such patients have negative biopsies, the raised PSA instead being due to, for example, prostate enlargement, prostatitis, or benign prostatic hyperplasia (BPH) [4]. Moreover, the clinical significance of many diagnosed low-grade $\mathrm{PCa}$ is questionable, and this issue represents a challenge in the current management of PCa. New clinical assays are accordingly required to reduce over detection and improve early detection of significant tumors [6]. A diagnostic tool capable of distinguishing patients with clinically significant cancer who need curative treatment from those with indolent cancer who would benefit from active surveillance is an urgent need.

The PCA3 non coding mRNA is a molecular biomarker with high specificity for PCa that can be determined in urine of patients with suspected PCa. It represents an additional diagnostic test that reduces the indications for biopsy and improves its efficiency [7]. Moreover, PCA3 could help to avoid over detection of clinically insignificant PCa while not missing the detection of clinically significant cancer as it has been associated with characteristics indicative of tumor aggressiveness such as tumor volume, tumor grade, and Gleason score [8-10]. Used in conjunction with established imaging modalities and serological and clinical data, PCA3 may also be a useful marker to improve the selection of patients suitable for active surveillance or focal therapy.

The purpose of this study was to evaluate the yield of PCA3 as an additional test in the management of patients with suspected $\mathrm{PCa}$ and to investigate whether our previously published results, in a prospective study of 598 patients, would be confirmed [11]. Moreover, the association of PCA3 with the new Grade Groups proposed by the International Society of Urological Pathology (ISUP) in 2014 was evaluated [12-14].

\section{Patients and Methods}

This retrospective study included data from consecutive patients with suspected $\mathrm{PCa}$ who were candidates for prostate biopsy and who presented to the urology office between November 2009 and April 2016. A protocol for the study, including the inclusion and exclusion criteria, and a copy of the informed consent form to be signed by patients were approved by the Ethics Committee of our institution.

\section{Samples and Patients}

A total of 1038 urine samples collected after prostate massage were studied in our laboratory within the period stated above. In order for samples to be included in this study, sample collection had to be indicated by one of the following: elevated PSA and prior negative biopsy, altered PSA but lower than $10 \mathrm{ng} / \mathrm{ml}$ without prior biopsy, altered PSA and presence of a well-known prostatic inflammatory disease (prostatitis or BPH), or high PSA level in the presence of a co morbidity giving rise to an increased biopsy-associated risk. Samples corresponding to patients with previous PCa diagnoses (71) were excluded.

A total of 967 urine samples corresponding to 814 patients were included. One hundred and thirty-two patients had more than one sample studied during their clinical follow-up. When comparative statistical analysis with other clinical variables (age, serum PSA, presence of prostatitis, and prostate volume) was performed, only the latest study data were used, resulting in the exclusion of 153 urine samples. Data recorded included: age, total PSA $(\mathrm{ng} / \mathrm{ml})$, and prostate volume (cc) of all patients. When a biopsy was performed, the number of cylinders obtained was also recorded, and when a PCa was diagnosed, the number of affected cylinders, the percentage of tumor, the Gleason score, and the Grade Group were recorded as well. In those cases diagnosed as PCa before publication of the Gleason Grade Group guidelines, the Gleason score was converted to its corresponding Grade Group according to published instructions [12-14].

\section{PCA3 Determination}

The first voided urine after DRE with prostatic massage consisting in three palpations per lobe was collected and tested with the Progensa ${ }^{\mathrm{TM}}$ PCA3 Assay kit (Gen Probe) following the manufacturer's instructions. The kit quantifies PCA3 and PSA mRNA molecules and generates a PCA3 score(s-PCA3) according to the formula: (PCA3 mRNA/PSA mRNA) $\times 1000$. The s-PCA3 was considered positive when it was 35 or higher and only in these cases was a prostate biopsy recommended owing to the high probability of detecting PCa [15]. When the association 


\section{Open Access Journal of Cancer \& Oncology}

between PCA3 and the parameters of tumor aggressiveness was analyzed, the s-PCA3 cut-off was established at 50, in accordance with previous studies $[11,16,17]$.

\section{Prostate Biopsy Protocol}

Tran rectal ultrasound-guided prostate biopsies were performed in an operating room with patients under anesthesia with sedation. A minimum of five cylinders per lobe were obtained, with additional cores when suspicious nodules were detected by DRE or ultrasound. In those patients who had a previous negative biopsy, a minimum of ten cylinders per lobe were obtained.

\section{Histopathological Study}

Two pathologists performed the histopathological study on serial sections of formalin-fixed paraffinembedded tissue stained with hematoxylin-eosin. Immunohistochemical study with alpha ethylacyl-CoAracemase (AMACR) and basal cell markers (p63 and $34 \beta E 12$ cytokeratins) was performed when requested by the pathologist. The diagnostic entities covered were: prostate adenocarcinoma (PCa), atypical small acinar proliferation (ASAP), high-grade prostatic intraepithelial neoplasia (HGPIN), chronic prostatitis, other nonneoplastic processes, and normality. In $\mathrm{PCa}$ cases the grade was determined based on the Gleason patterns and the Grade Group [12-14,18]. The new ISUP grading system for prostatic adenocarcinoma classifies PCa into five prognostically distinct Grade Groups (1-5) [12]. This classification reflects prostate cancer biology more accurately than does the Gleason score. The Grade Group 1 comprises patients with Gleason $(3+3) \mathrm{PCa}$, mostly with an excellent prognosis and no potential lymph node involvement; such patients are suitable for active surveillance although other clinical information must be considered in the decision on the best treatment [19].

The ASAP category was reserved for micro glandular proliferations that displayed morphological and immune histo chemical characteristics suspicious for PCa (loss of basal layer and/or expression of AMACR) but were insufficiently represented in biopsies to support a definitive diagnosis of PCa.

In those cases diagnosed as PCa, the presence of tumor in more than $33 \%$ of the cylinders was considered an indicator of tumor stage.

\section{Statistical Analysis}

Hypothetical associations between pairs of variables were analyzed using Fisher's exact test for the categorical variables and linear regression analysis for the continuous variables. To determine the association between a shortlist of three categorical variables, the best log-linear model was established using the stepwise algorithm and the Akaike information criterion starting from the full model, i.e. with all possible interactions among the three variables [20]. In order to investigate whethers-PCA3 could predict the Grade Group, a logistic model was applied [21]. All statistical analyses were performed with the R software [22].

\section{Results}

A total of 967 urine samples corresponding to 814 patients with a mean age of 65.8 years (SD 8.1 years) were included. Most of the s-PCA3tests (91.7\%) were performed in patients with elevated PSA and a negative previous biopsy or alterations in PSA levels not exceeding $10 \mathrm{ng} / \mathrm{ml}$ (Table 1). A valid result was obtained in $98.5 \%$ of samples. The means-PCA3value was 48.7. Details of the numeric variables analyzed are reported in Table 2 . The s-PCA3showed a very weak significant relationship with age ( $<<0.05$, with $5.4 \%$ of the variability explained by the s-PCA3) and no significant relation with serum PS A level $(\mathrm{p}=0.798)$, presence of prostatitis $(\mathrm{p}=0.826)$, or prostate volume $(\mathrm{p}=0.130)$. Five hundred and twenty-two samples corresponding to 431 patients had a negative s-PCA3. In these cases, accounting for $54 \%$ of the samples and $52.9 \%$ of patients, no biopsy was indicated. Nevertheless, a biopsy was performed in 37 patients with a negative sPCA3 following the urologist criterion. Twenty-four (5.6\%) patients had a negative biopsy, of whom two $(0.5 \%)$ were diagnosed with HGPIN, one $(0.2 \%)$ with ASAP, and ten $(2.3 \%)$ with PCa.

\begin{tabular}{|c|c|}
\hline Indication for the s-PCA3 study & No. of samples (\%) \\
\hline Negative prior biopsy & $467(48.3)$ \\
\hline Elevated PSA $\leq 10 \mathrm{ng} / \mathrm{ml}$ & $420(43.4)$ \\
\hline Risk factors for biopsy & $30(3.1)$ \\
\hline Known benign prostate pathology & $27(2.8)$ \\
\hline No specified indication & $23(2.4)$ \\
\hline Total & $967(100)$ \\
\hline
\end{tabular}

Table 1: Indications for study of the PCA3 score (s-PCA3).

\begin{tabular}{|c|c|}
\hline & $\begin{array}{c}\text { Median } \\
\text { (minimum-maximum) }\end{array}$ \\
\hline Total PSA (ng/ml) & $5.8(0.5-134)$ \\
\hline Prostate volume (cc) & $50(2-200)$ \\
\hline Percentage of affected cylinders & $20(1-100)$ \\
\hline
\end{tabular}

Table 2: Description of the variables studied.

In patients with samples that had a positive s-PCA3 $(44.5 \%)$, a subsequent biopsy was recommended. Of the 
151 biopsies studied, $56.3 \%$ yielded a diagnosis of $\mathrm{PCa}$, 4.6\% ASAP, and 6\% HGPIN, while 33.1\%showed no evidence of malignancy. When the s-PCA3cut-off was set to 50 instead of 35 , the percentage of PCa increased to $59.5 \%$ and the percentage of biopsies with no evidence of malignancy decreased to $30.2 \%$, while the percentages of ASAP and HGPIN did not change significantly. The probability of a positive biopsy increased as the $\mathrm{s}$-PCA3increased $(\mathrm{p}=0.041)$.The percentage of cylinders with carcinoma detected in the biopsy was assessed in 75 of the 85 cases with a diagnosis of PCa; the ten remaining cases were surgical resection specimens. The percentage of affected cylinders increased as the s-PCA3increased, the relationship being statistically significant $(\mathrm{p}=0.015)$. In no patient with a diagnosis of $\mathrm{PCa}$ and ans-PCA3 between 35 and 50 were more than $33 \%$ of cylinders affected (Figure 1). The s-PCA3 and the Gleason score showed a statistically significant relationship $(\mathrm{p}=0.001)$. Of those cases with ans-PCA3 greater than $50,76.8 \%$ had a Gleason score $\geq 7$ (Figure 2). The best log-linear model, including s-PCA3, the Gleason score, and the percentage of affected cylinders, retained in the final equation the interactions between s-PCA3 and percentage of affected cylinders $(\mathrm{p}=0.002)$ and between s-PCA3 and the Gleason score $(\mathrm{p}<0.000)$, confirming the relationship shown previously with Fisher's exact test.

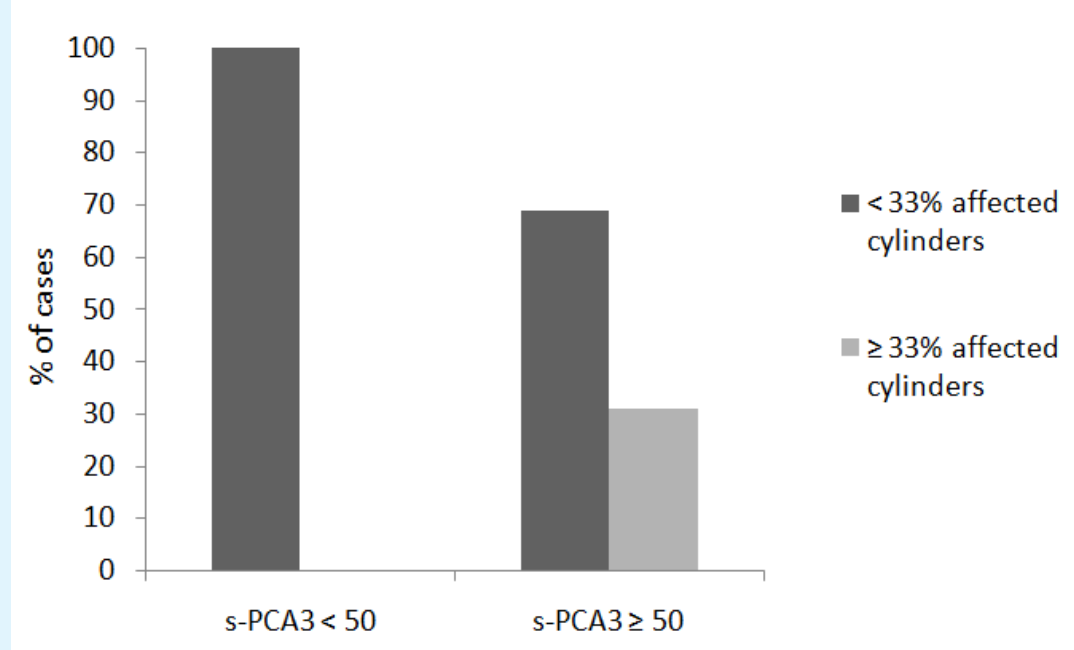

Figure 1: Percentage of affected cylinders in the biopsy in relation to the PCA3 score (s-PCA3) in patients with a diagnosis of PCa $(n=75)$.

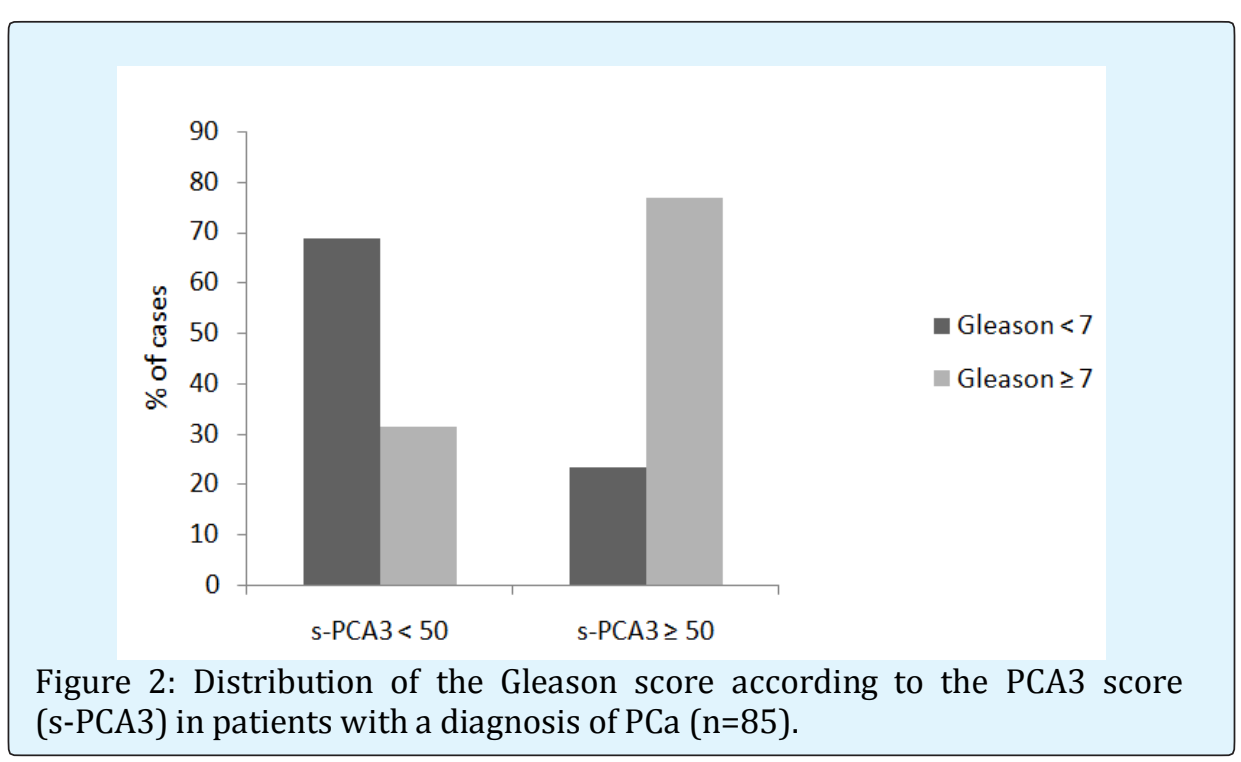

Only one patient in this series was in Grade Group5. For the purpose of all statistical analyses, this patient was grouped with those in Grade Group4.A statistically significant relationship was observed betweens-PCA3 and the Grade Group ( $\mathrm{p}=0.008)$. Of PCa patients with a positive s-PCA3 lower than 50,68.8\% were in Grade 
Group1 (Figure 3). The best log-linear model, including sPCA3, the Grade Group, and the percentage of affected cylinders, retained in the final equation the interactions between s-PCA3and percentage of affected cylinders $(p=0.002)$ and between $s-P C A 3$ and the Grade Group $(p=0.003)$, confirming the relationship shown previously with Fisher's exact test (Figure 4). Alogistic model was employed to test whether s-PCA3and the percentage of affected cylinders could predict the Grade Group. A statistically significant relationship between the three variables was confirmed, as was shown previously by Fisher's exact test and the log-linear model. At a cut-off point of 0.625 , the sensitivity and specificity of the model were $79 \%$ and $64 \%$ respectively; the positive predictive value was $84 \%$ and the negative predictive value was $56 \%$.

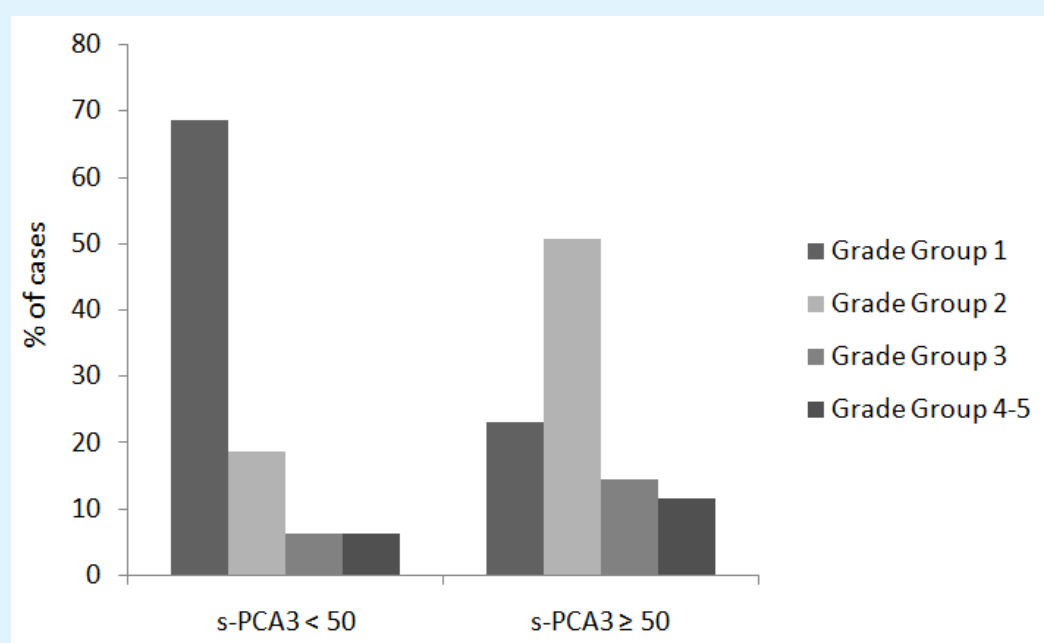

Figure 3: Distribution of the Grade Group according to the PCA3 score (s-PCA3) in patients with a diagnosis of $\mathrm{PCa}(\mathrm{n}=85)$.

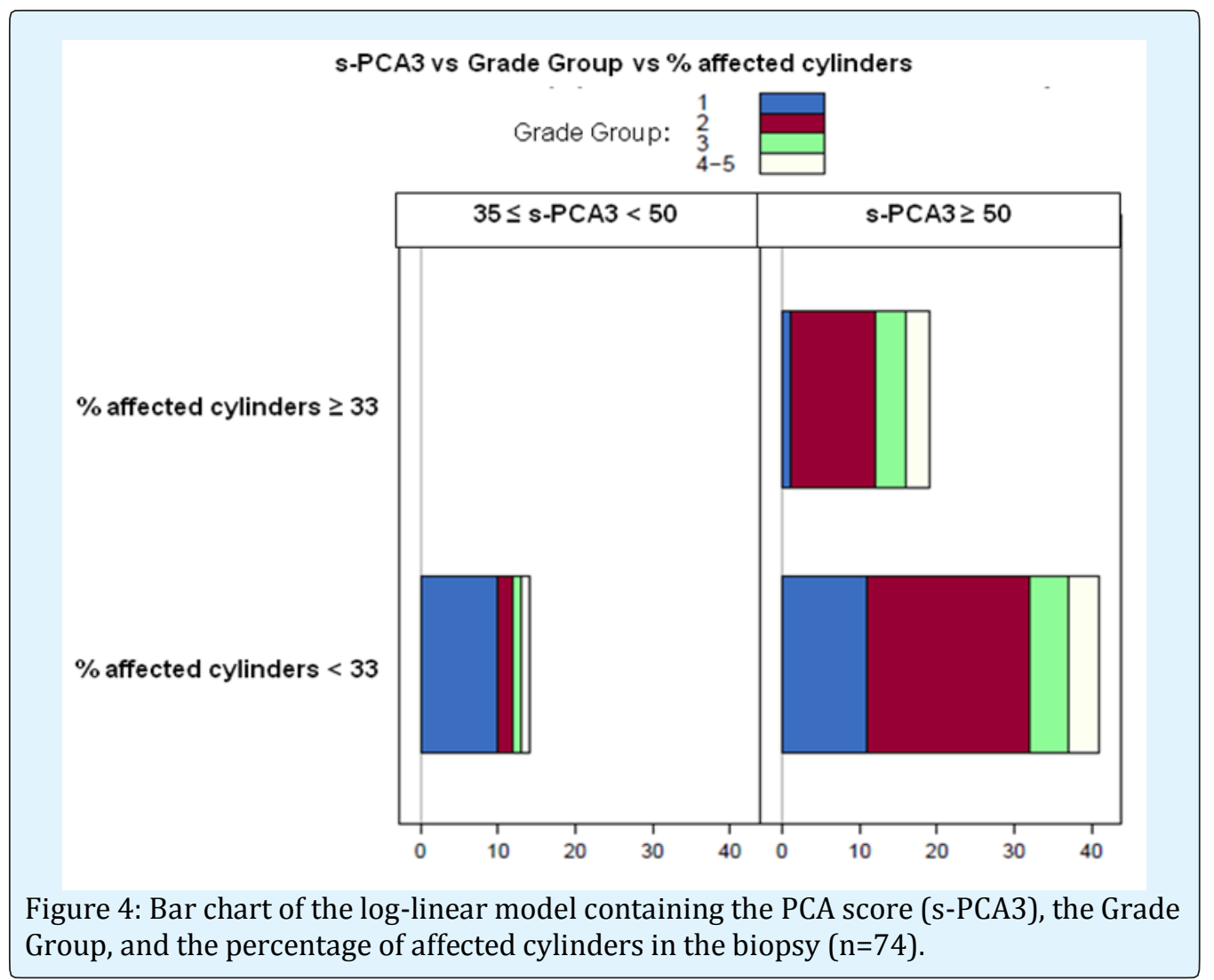




\section{Open Access Journal of Cancer \& Oncology}

\section{Discussion}

In this series the s-PCA3 was not related to PSA level, prostate volume, or presence of prostatitis, in agreement with data published in previous studies [23-25]. Moreover, only a very weak significant relationship was found with patient age. Incorporation of the PCA3 study into PCa screening significantly reduced (by 54\%) the indication for biopsy, in accordance with previously published data $[11,17,26,27]$. In patients with a positive sPCA3, the percentage of positive biopsies was markedly increased (to $60.9 \%$, including ASAP) compared with the percentage in biopsies indicated by PSA and DRE alone. There was a statistically significant relationship between s-PCA3 and the presence of PCa in the subsequent biopsy, as has been reported previously $[16,25,28]$. The s-PCA3 was not able to predict the presence of PCa in ten patients in the series. This percentage of false negative results is in accordance with that reported by the PCA3 kit manufacturer. The relation between s-PCA3 and different parameters associated with tumor aggressiveness, such as Gleason score and the percentage of cylinders in which PCa was detected, was also analyzed. All of the latter parameters showed a statistically significant relationship with s-PCA3. None of the patients with a positive s-PCA3 below 50 showed more than $33 \%$ of cylinders affected by PCa and $68.8 \%$ of them had a Gleason score below 7. In contrast, $76.8 \%$ of patients with ans-PCA3 over 50 had a Gleason score higher than or equal to 7.These results are in agreement with previously published data from reviews and meta-analyses and also confirm our previous results $[11,16,28,29]$. To our knowledge this is the first time that the relation between s-PCA3 and the new Grade Group has been explored. In this series there was a statistically significant relationship between s-PCA3 and the Grade Group. These results were confirmed in two log-linear models. The high prevalence of low Grade Group tumors in patients with PCa and a positive PCA3 lower than 50 may warrant recommendation of a conservative clinical attitude in this subgroup. A logistic model was designed in order to analyze whether s-PCA3 could predict the Grade Group in patients with PCa. This model once more confirmed a strong relationship between s-PCA3 and the Grade Group. However, its predictive capacity was weak, probably owing to the small size of the series, and more data are needed in order to confirm these preliminary results. In our series, $68.6 \%$ of PCa with a positive s-PCA3 below 50 were in Grade Group 1, indicating that subsequent biopsy could be avoided or delayed in this patient subgroup, with a watchful waiting strategy instead being adopted unless contraindicated by other clinical information. In contrast, the $76.8 \%$ of patients in our series with an s-PCA3 higher than 50 were assigned to Grade Groups $2-5$, suggesting

Rodon N, et al. A Low Score in Patients with a Positive PCA3 Urine Test Predicts the Lowest Prognostic Category in the New Grading System for Prostate Carcinoma. J Cancer Oncol 2018, 2(1): 000116. that a more aggressive therapeutic approach may be adequate. Considering all the results, in addition to the new evidence showing that a significant relationship between s-PCA3 and the Grade Group, it was confirmed that, as suggested by previous studies $[11,17,28,29]$. SPCA3 has prognostic significance in prediction of the aggressiveness of PCa $[11,17,28,29]$.

\section{Conclusions}

In this retrospective study we confirmed our previously published results showing that s-PCA3outperforms PSA in predicting biopsy diagnosis of $\mathrm{PCa}$. Moreover, the prognostic significance of s-PCA3 was confirmed, as it was associated with parameters of tumor aggressiveness such as tumor volume and Gleason score. Importantly, to our knowledge this is the first time that an association has been demonstrated between s-PCA3 and the new Grade Group.In conclusion, s-PCA3 may serve as an additional marker to reduce the indication for biopsies andto avoid overdiagnosis and overtreatment of patients with suspected PCa. Moreover, insofar as it is predictive of the Grade Group and tumor extension, s-PCA3 can provide information of prognostic significance.

\section{Acknowledgment}

To Eva Torija, Montse Alcaraz, Ana Guardiola and Cristina García for her secretarial assistance.To Olga Díaz, BegoñaMartínez, Nuria Arraiza, Sara Rivas and VerónicaSerrano for her technical assistance.

\section{References}

1. (2014) World Cancer Report. International Agency for Research on Cancer, ISBN 978-92-832-0429-9.

2. Squire JA, Yoshimoto M, Alami J, Park PC, Williams JL, et al. (2011) Prostate cancer as a model system for genetic diversity in tumors. Adv Cancer Res 112: 183213.

3. Schöder FH, Wildhagen MF (2001) Screening for prostate cancer: evidence and perspectives. BJU International 88(8): 811-817.

4. Schroder FH, Hugosson J, Roobol MJ, Tammela TL, Ciatto S, et al. (2009) Screening and prostate-cancer mortality in a randomized European study. N Engl J Med 360(13): 1320-1328.

5. Andriole GL, Crawford ED, Grubb RL, Buys SS, Chia D, et al. (2009) Mortality results from a randomized prostate-cancer screening trial. N Engl J Med 360(13): 1310-1319. 


\section{Open Access Journal of Cancer \& Oncology}

6. Hamdy FC, Donovan JL, Lane JA (2016) 10-year outcomes after monitoring, surgery or radiotherapy for localized prostate cancer. N Engl J Med 375: 14151424.

7. Wei JT, Feng Z, Partin AW, Brown E, Thompson I, et al. (2014) Can urinary PCA3 supplement PSA in the early detection of prostate cancer? J ClinOncol 32(36): 4066-4072.

8. Whitman EJ, Groskopf J, Ali A, Chen Y, Blase A, et al. (2008) PCA3 score before radical prostatectomy predicts extracapsular extension and tumor volume. J Urol 180(5): 1975-1978.

9. Nakanishi H, Groskopf J, Fritsche HA, Bhadkamkar V, Blase A, et al. (2008) PCA3 molecular urine assay correlates with prostate cancer tumor volume: implication in selecting candidates for active surveillance. J Urol 2008 179(5): 1804-1809.

10. Merola R, Tomao L, Antenucci A, Sperduti I, Sentinelli S, et al. (2015) PCA3 in prostate cáncer and tumor aggressiveness detection on 407 high-risk patients: a National Cancer Institute experience. J Exp Clin Cancer Res 34(1): 15.

11. Rodon N, Trias I, Verdu M, Román R, Domínguez A, et al. (2014) Diagnostic and predictive value of urine PCA3 gene expression for the clinical management of patients with altered prostate-specific antigen. Actas UrolEsp 38(3): 150-155.

12. Epstein JI, Egevad LE, Amin MB, Delahunt B, Srigley JR, et al. (2016) The 2014 International Society of Urological Pathology (ISUP) consensus conference on Gleason grading of prostatic carcinoma.Definition of grading patterns and proposal for a new grading system. Am J SurgPathol 40(2): 244-252.

13. Loeb S, Montoris F, Catto JW (2015) Future proofing Gleason grading: What to call Gleason 6 prostate cancer?.Eur Urol 68(1): 1-2.

14. Kryvenko ON, Epstein JI (2016) Changes in prostate cancer grading: Including a new patient-centric grading system. Prostate 76(5): 427-433.

15. Groskopf J, Aubin SM, Deras IL, Blase A, Bodrug S, et al. (2006) APTIMA PCA3 molecular urine test: development of a method to aid in the diagnosis of prostate cancer. Clin Chem 52(6): 1089-1095.

16. Ruiz-Aragón J, Márquez-Peláez S (2010) Assesment of the PCA3 test for prostate cancer diagnosis: a systemic review and meta-analysis. Actas UrolEsp 34(4): 346-355.

17. Rodon N, Trias I, Verdu M (2014) PCA3 gene in prostate diagnosis: Improving biopsy efficiency and predicting tumor grade and extension. Modern Pathol 27: $255 \mathrm{~A}$.

18. Epstein JI, Allsbrook WC Jr, Amin MB, Egevad LL (2005) The 2005 International Society of Urological Pathology (ISUP) Consensus Conference on Gleason Grading of Prostatic Carcinoma. Am J Surg Pathol 29(9): 1228-1242.

19. Ross H, Kryvenko ON, Cowan JE, Simko JP, Wheeler TM, et al. (2012) Do adenocarcinomas of the prostate with Gleason score $\leq 6$ have the potential to metastasize to lymph nodes?. Am J Surg Pathol 36(9): 1346-1352.

20. Friendly M (2017) Working with categorical data with R and the vcd and vcd Extra packages.

21. Verzani J (2004) Using R for introductory statistics. Chapman \& Hall/CRC, US.

22. R Core team (2016) R: A language and environment for statistical computing. R Foundation for Statistical Computing, Vienna, Austria.

23. De Luca S, Passera R, Milillo A, Coda R, Randone DF (2012) Histological chronic prostatitis and high-grade prostate intra-epithelial neoplasia do not influence urinary prostate cancer gene 3 score. BJU Int 110(11 Pt B): E778-82.

24. Haese A, de la Taille A, van Poppel H, Marberger M, Stenzl A, et al. (2008) Clinical utility of the PCA3 urine assay in European men scheduled for repeat biopsy. Eur Urol 54: 1081-1088.

25. de la Taille A, Irani J, Graefen M, Chun F, de Reijke T, et al. (2011) Clinical evaluation of the PCA3 assay in guiding initial biopsy decisions. J Urol 185(6): 21192125.

26. Hessels D, Schalken JA (2009) The use of PCA3 in the diagnosis of prostate cancer. Nat Rev Urol 6(5): 255261.

27. GittelmanM , Hertzman B, Bailen J, Williams T, Koziol I, et al. (2013) PROGENSA® PCA3 molecular urine test as a predictor of repeat prostate biopsy outcome in men with previous negative biopsies: A prospective multicenter clinical study. J Urol 190(1): 64-69. 


\section{Open Access Journal of Cancer \& Oncology}

28. Chevli KK, Duff M, Walter P, Yu C, Capuder B, et al. (2014) Urinary PCA3 as a predictor of prostate cancer in a cohort of 3.073 men undergoing initial prostate biopsy. J Urol 191(6): 1743-1748.
29. van Poppel H, Haese A, Graefen M, de la Taille A, Irani J, et al. (2012) The relationship between Prostate Cancer gene 3 (PCA3) and prostate cancer significance. BJU Int 109(3): 360-366. 